\title{
GPPS-BJ-2019-028
}

\section{A physical model of flow-induced acoustic resonance in a duct containing a circular cylinder}

\author{
Zhiliang Hong \\ College of Airworthiness, \\ Civil Aviation University of China \\ zlhong@cauc.edu.cn \\ Tianjin, PR China
}

\author{
Mingsui Yang \\ The $11^{\text {th }}$ Research Department, \\ AECC Shenyang Engine Research Institute \\ yangmingsui@126.com \\ Shenyang, Liaoning, PR China
}

\begin{abstract}
A physical model to study the acoustic resonance induced by the wake flow of a cylinder in a flow duct is established. This model consists of three meshless submodels operated in the time domain, including the discrete vortex method (DVM) for vortex shedding, the vortex sound theory for sound radiation and the time-domain boundary element method (TDBEM) for sound propagation within the duct. In addition, the acoustic particle velocity is calculated to take the sound feedback effect into consideration, which makes the coupling process between flow and sound field to be bi-directional. The distribution patterns of vortex street behind the cylinder and the acoustic $\beta$-mode within the duct are reasonably depicted. And both the qualitative and quantitative results of resonant frequency and amplitude can be fully compared with the previous experiment data, especially, the lock-in phenomenon is well captured. The lock-in regime depicts as a competition between the vortex shedding frequency $\left(f_{\mathrm{s}}\right)$ and the inherent frequency of $\beta$-mode $\left(f_{\mathrm{a}}\right)$. And two lock-in patterns, the synchronous regime and $\beta$ mode dominant regime, are identified according to the mismatch between $f_{\mathrm{s}}$ and $f_{\mathrm{a}}$. This physical model provides the proper characteristics of acoustic resonance as observed in experiment, and helps to get a more detailed understanding of the lock-in mechanism.
\end{abstract}

\section{INTRODUCTION}

Aeolian tone, caused by vortex behind bluff bodies in flow, has received continuous attention since 1870s. And it is well known that the frequency of aeolian tone is always identical with the vortex shedding frequency, which varies linearly with the flow speed in the free space. However, if a bluff body is placed in a confined space, such as a flow duct, the sound generation process becomes much more complicated, because of the fierce vortex sound interaction under proper conditions. This phenomenon is recognized as acoustic resonance, which is usually accompanied with unique high sound pressure level (SPL, even over 170dB). It cannot only cause severe noise pollution issues but also lead to performance deterioration or even catastrophic damage to the facility. These dangers, undoubtedly, should be avoided in various military and civilian engineering applications, such as combustion chambers of rocket (Karthik et al., 2008), compressors of aeroengine (Parker, 1968; Liu et al., 2018), front grills of automobile (Yokoyama, 2013), tube banks of heat exchanger (Shaaban and Mohany, 2018) and so forth. Consequently, the relevant researches are greatly motivated.

Although the general process of acoustic resonance has been proposed, the underlying mechanism of vortex sound interaction is still an open issue. To explore the underlying mechanism, a simplified system like a single cylinder in a flow duct is preferred instead of the complicated situations occurring in the above-mentioned engineering applications.

Acoustic resonance in a duct induced by wake flow from an inner circular cylinder has been widely studied since the 1950s, and the research progresses are summarized in some review papers (Blevins, 1984; Mohany, 2012, etc.). Through detailed flow visualization measurement, the high correlation between resonant sound and wake vortex shedding process has been substantiated. On one hand, when an intense tone noise is excited, the vortex shedding becomes highly coherent in the streamwise direction and forms a succession of vortex clouds. On the other hand, if the wake flow is disturbed by turbulent devices, such as small rods, the resonance could be fully eliminated (Katasonov et al., 2015). A thorough investigation on the vortex sound interaction would do benefit to the basic understanding of the acoustic resonance mechanism. Certain progress has been made in this issue with the help of numerical methods, however, it is far from satisfactory, as how to simulate the coupling between vortex and sound is still a difficult task even in nowadays with the highly developed computer science, due to the large difference in spatial scale of the unsteady flow and sound variations. Most of the previous researches are based on the decoupling method, and the effect of feedback 
sound on vortex shedding is simulated through artificial external sound excitation (Tan et al., 2005; Mohany and Ziada, 2009; Mohany, 2012; Mohany et al., 2014; Arthurs et al., 2013) or oscillation (Davies, 1976; Lotfy and Rockwell, 1993). These studies help to depict the effect of sound on the character of wake vortex, such as the vortex formation length, convection velocity and circulation of the vortex, and can also give a qualitative analysis to the energy transfer from sound to flow field. As vortex sound interaction occurs bidirectionally and spontaneously, however, artificially external sound excitation is unidirectional, which obviously deviates from the actual situation and prevents the prediction of resonant amplitude. In this aspect, the above decoupling method is not satisfactory for exploring the underlying mechanism of vortex sound interaction, which is the main motivation of present study.

In this work, we aim to establish a closed physical model that calculates the unsteady flow field, sound field and their interaction simultaneously. To this end, the meshless methods are used for efficiency solution. Firstly, the discrete vortex method is adopted to simulate the wake of cylinder, and then a sub-model corresponding to the sound pressure radiated from vortex shedding is derived based on vortex sound theory. Next, the time domain boundary element method is introduced to calculate the sound field within the duct, which involves the reflection and transmission effects of the rigid wall and the ends of the duct. In the end, the sound feedback effect is realized through adding acoustic particle velocity, calculated based on the sound pressure results, onto the vortex shedding process to close the entire model. It is expected that results of the present model can help to improve the understanding of acoustic resonance in the following aspects: (a) the variation of sound pressure and frequency with increasing flow velocity, (b) the distribution of the flow and sound fields within the duct, (c) the effect of feedback sound on the flow field.

\section{MODELING PROCEDURE}

As is known, to depict the unsteady coupling process, a time-domain method would be preferred. With the limitation of the huge time consumption for unsteady flow and sound field solution, it is really a great challenge to conduct the solution in three-dimensional. Besides, as the dimensions of a cylinder in the spanwise is usually much larger than that in the streamwise, it is reasonable to make a two-dimensional (2D) assumption for convenience.

\section{DVM for the unsteady wake flow of a 2D circular cylinder}

As shown in Fig. 1, a circular cylinder with a radius of $a$ is immersed in the incompressible uniform flow $U$. The DVM employed here is based on the original work proposed by Sarpkaya and Schoaff (1979), and only the main aspects are introduced here for conciseness.

The incoming flow is assumed to be irrotational except at the places where a point vortex locates, and in addition, it is inviscid except at the two points where the vortices separated from the cylinder, as illustrated in Fig. 1 with a separation angel of $\pm \theta_{s}$.

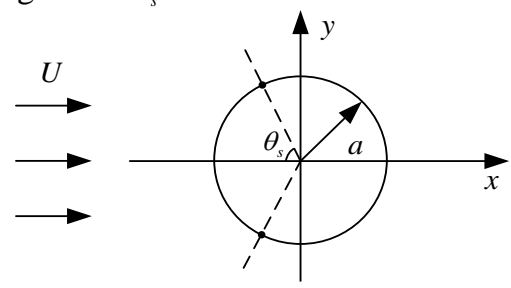

Figure 1. Schematic Diagram of the Flow Model.

As the flow field is assumed to be inviscid, the strengths of the shed vortices should be kept constant. However, an attenuation function is used to artificially simulate the viscous effect (Kiya et al., 1982), which is expressed as

$$
\frac{\Gamma(t)}{\Gamma_{0}}=1-\exp \left(-\frac{A}{4(U t) / a}\right),
$$

where $\Gamma_{0}$ and $\Gamma(\mathrm{t})$ are the strength at initial time and time $t$ of a point vortex respectively, $A$ is a constant which only relates to the type of the incoming flow, and it is set to be 32 based on numerical trials.

The Kelvin's theorem is used to determine the trajectory of a point vortex, after shedding into the flow field

$$
\frac{\mathrm{d} \mathbf{z}_{n}}{\mathrm{~d} t}=\mathbf{w}\left(\mathbf{z}_{n}\right)
$$

where $\mathbf{z}_{\mathrm{n}}$ is the complex coordinate of the $n$th point vortex, $\mathbf{w}\left(\mathbf{z}_{\mathrm{n}}\right)$ represents the complex velocity, expressed as

$$
\mathbf{w}\left(\mathbf{z}_{n}\right)=U-\frac{U a^{2}}{\mathbf{z}^{2}}+\frac{\Gamma_{n}}{2 \pi i} \sum_{n=1}^{N}\left[\frac{1}{\mathbf{z}-\mathbf{z}_{n}}-\frac{1}{\mathbf{z}-a^{2} / \overline{\mathbf{z}}_{n}}\right],
$$

where $N$ is the total number of the vortices in the wake. To satisfy the rigid wall condition on the cylinder surface, the complex potential is obtained by placing an image vortex with strength $-\Gamma_{n}$ at the inverse point $\mathbf{z}=a^{2} / \overline{\mathbf{z}}_{n}$, where the bar denotes the complex conjugate. Besides there is no image vortex at the centre of the cylinder to ensure that the total circulation in the whole domain vanishes.

Although the separation point oscillates periodically due to the upstream influence from the wake, quite reasonable results can be obtained if the simpler assumption is made of fixed separation points (Lewis, 1992). Therefore, in this model the separation is supposed to occur at the fixed positions at $\theta_{\mathrm{s}}= \pm 78^{\circ}$ from the front stagnation point. At the beginning of each time step, a point vortex is shed from the upper and lower separation point respectively, with the strength $\Gamma_{\mathrm{nv}}$ being determined by

$$
\Gamma_{n v}=0.5 U_{s}^{2} \Delta t
$$

where $U_{\mathrm{s}}$ is the outer velocity of the shear layer at the separation point, and the thickness of the shear layer is empirically set to be $0.01 a$. To satisfy the no-slip condition at the separation point, the position of the nascent vortex is determined by 


$$
\mathbf{z}_{\mathrm{nv}}=a\left[\frac{1+\left|\Gamma_{\mathrm{nv}}\right| /\left(2 \pi U_{\mathrm{s}} a\right)}{1-\left|\Gamma_{\mathrm{nv}}\right| /\left(2 \pi \mathrm{U}_{\mathrm{s}} a\right)}\right] \exp \left[i\left(\pi-\theta_{\mathrm{s}}\right)\right] .
$$

The aerodynamic force on the cylinder is given by

$$
C_{\mathrm{d}}+i C_{1}=i \frac{\partial}{\partial t} \sum_{n=1}^{N_{\mathrm{v}}} \Gamma_{n}\left(\mathbf{z}_{n}-a^{2} / \overline{\mathbf{z}}_{n}\right)
$$

where $C_{\mathrm{d}}$ and $C_{\mathrm{l}}$ is the drag and lift coefficient respectively, and $N_{\mathrm{v}}$ is the total number of point vortices in the flow field.

\section{Vortex sound theory for the sound radiation from the shed vortices in low Mach number flow}

In a low Mach number, isentropic and inviscid flow field, the sound wave equation is expressed as (Powell, 1964)

$$
\left(\frac{1}{c_{0}^{2}} \frac{\partial^{2}}{\partial t^{2}}-\nabla^{2}\right) \frac{p}{\rho_{0}}=\nabla \cdot(\boldsymbol{\omega} \times \mathbf{v}),
$$

where $c_{0}$ is the velocity of sound wave, $p$ is the sound pressure, $\rho_{0}$ is the ambient far-field density, $\omega$ is the vorticity of a vortex, and $\mathbf{v}$ is the convection velocity of the vortex.

In the 2D free space, the general solution of sound pressure induced by this vortex can be expressed as

$$
\frac{p_{n}}{\rho_{0}}=-\int_{0}^{t} \int_{S}\left(\omega_{n} \times \mathbf{v}_{n}\right) \cdot \nabla G d S d \tau,
$$

where $\boldsymbol{\omega}_{n}$ is the vorticity of the $n$th vortex, $\boldsymbol{\omega}_{n}=\mathbf{k} \Gamma_{n} \delta\left(x-x_{n}(\tau)\right) \delta\left(y-y_{n}(\tau)\right), \quad \mathbf{v}_{n} \quad$ is the convection velocity of the vortex, $\mathbf{v}_{n}=\mathbf{i} v_{n x}+\mathbf{j} v_{n y}$, and $G$ is the 2D free space Green's function in the time domain, expressed as

$$
G(\mathbf{x}, t / \mathbf{y}, \tau)=\frac{c_{0} H\left[c_{0}(t-\tau)-R\right]}{2 \pi \sqrt{c_{0}^{2}(t-\tau)^{2}-R^{2}}},
$$

where $R$ is the distance between the source $\mathbf{y}\left(y_{1}, y_{2}\right)$ and receiver $\mathbf{x}\left(x_{1}, x_{2}\right), H$ is the Heaviside function, $t$ is the receipt time and $\tau$ is the emission time.

According to (8) and the expression of $\boldsymbol{\omega}_{n}$, we have

$$
\frac{p_{n}}{\rho_{0}}=\left.\int_{0}^{t} \Gamma_{n} v_{n y} \frac{\partial G}{\partial y_{1}}\right|_{\left(x_{n}(\tau), y_{n}(\tau), \tau\right)} d \tau-\left.\int_{0}^{t} \Gamma_{n} v_{n x} \frac{\partial G}{\partial y_{2}}\right|_{\left(x_{n}(\tau), y_{n}(\tau), \tau\right)} d \tau
$$

The two partial derivative terms are expressed as

$$
\frac{\partial G}{\partial y_{1,2}}=\frac{\partial R}{\partial y_{1,2}}\left[\frac{c_{0} R H\left[c_{0}(t-\tau)-R\right]}{2 \pi \sqrt{\left[c_{0}^{2}(t-\tau)^{2}-R^{2}\right]^{3}}}+\frac{\frac{\partial}{\partial \tau} H\left[R-c_{0}(t-\tau)\right]}{2 \pi \sqrt{c_{0}^{2}(t-\tau)^{2}-R^{2}}}\right] .
$$

After that, the sound pressure radiated from the $n$th vortex becomes

$$
\begin{gathered}
\frac{p_{n}}{\rho_{0}}=\int_{0}^{t}\left(X B+\frac{\partial X}{\partial \tau} \frac{G}{c_{0}}\right) d \tau, X=\Gamma_{n}\left(v_{n x} \frac{\partial R}{\partial y_{2}}-v_{n y} \frac{\partial R}{\partial y_{1}}\right), \\
B=\frac{c_{0}\left[c_{0}(t-\tau)-R\right] H\left[c_{0}(t-\tau)-R\right]}{2 \pi \sqrt{\left[c_{0}^{2}(t-\tau)^{2}-R^{2}\right]^{3}}} .
\end{gathered}
$$

For all vortices in the wake, the total sound pressure is

$$
p=\sum_{n=1}^{N_{\mathrm{v}}} p_{n} .
$$

\section{Image theory for the rigid wall condition on the cylinder surface}

The rigid wall condition should be applied on the cylinder surface during the sound propagation calculation. In this respect, the image theory is adopted, which means the presence of the wall is accounted for by image sound source. The parameters of the sound source (the $n$th point vortex) and its image are given in the flow field solution by DVM, as shown in Table 1.

Table 1. Parameters of sound source and its image

\begin{tabular}{ccc}
\hline Parameters & Sound source & Image source \\
\hline Strength & $\Gamma_{n}$ & $-\Gamma_{n}$ \\
Position & $\mathbf{z}_{n}$ & $a^{2} / \overline{\mathbf{z}}_{n}$ \\
Velocity & $u_{n}+i v_{n}$ & $\left(-u_{n}+i v_{n}\right) / \overline{\mathbf{z}}_{n}^{2}$ \\
\hline
\end{tabular}

\section{D TDBEM for sound field within the duct}

The integration expression of sound wave equation in the 2D duct can be written as (Mansur and Brebbia, 1982)

$$
4 \pi c(Q) p(Q, t)=\int_{0}^{t} \int_{\Pi}\left(G \frac{\partial p}{\partial \mathbf{n}}-p \frac{\partial G}{\partial \mathbf{n}}\right) \mathrm{d} \Pi d \tau+s
$$

where $\Pi$ is the boundary of the two-dimensional region $\Omega$, $\mathbf{n}$ is outward vector normal to the boundary, $c(Q)$ is a constant number related to the position of field point $Q, s$ is the sound source radiated from the vortices within the duct, as shown in (11).

Substituting (9) into (12), the following expression is finally derived

$$
c(Q) p(Q, t)=\int_{0}^{t} \int_{\Pi} b G \mathrm{~d} \Pi \mathrm{d} \tau+\int_{0}^{t} \int_{\Pi} \frac{\partial R}{\partial \mathbf{n}}\left(p B+\frac{\partial p}{\partial \tau} \frac{G}{c_{0}}\right) \mathrm{d} \Pi \mathrm{d} \tau+s .
$$

where $b=\partial p(Q, t) / \partial \mathbf{n}$

\section{Application of acoustic feedback to the vortex shedding}

According to Blevins's (1985) experimental results, it is the acoustic particle velocity rather than the sound pressure that influences the flow field. And the feedback process mainly takes place during the separation of vortex. Therefore, the acoustic particle velocity $\left(u_{\mathrm{a}}\right)$ near the separation point is added to $U_{\mathrm{s}}$ in Eqs. (4) and (5).

As the sound pressure within the duct has been obtained, then it is readily to determine the acoustic particle velocity through numerically solving the momentum equation

$$
u_{a}=-\frac{1}{\rho_{0}} \int \frac{\partial p}{\partial x} d t
$$




\section{NUMERICAL IMPLEMENTATION}

\section{Vortex shedding of the cylinder wake}

A typical time marching approach of flow field solution using DVM is implemented in the following four steps.

(1) The positions of nascent vortices shed from the upper and lower separation point at the start of time step $m$ are determined by Eq. (5).

(2) Assign the circulations of nascent vortices through Eq. (4), and then evaluate the circulations of previous vortices using Eq. (1).

(3) Update the positions of previous vortices existed in the flow field by solving Eq. (3) through the fourth order Runge-Kutta method.

(4) Increase the time step $t_{\mathrm{m}+1}=t_{\mathrm{m}}+\Delta t$, and then repeat the above three steps.

It should be stated that, the wake flow is smoothed by assuming a Rankine vortex velocity field, and the radius of vortex core is set to be $0.8 \Lambda$, where $\Lambda$ is the length of the feeding sheet of the nascent vortex (Hong et al., 2014). Besides, we employ two kinds of method to keep the total vortices within acceptable bounds. The first one is attenuation of the vortex circulation through Eq. (1), which means the vortices are gradually decreased in strength when they are convecting downstream with the increase of time steps. The second one is neglecting the effects of vortices blown a certain distance far away from the cylinder, and it is set to be $60 a$ in this study.

\section{Sound wave propagation within the duct}

The conventional collocation method is employed to discretize Eq.(13), which means the boundary $\Pi$ is represented by a set of discrete nodes $Q_{j}, j=1,2,3, \ldots$ and the time is discretized into continuous intervals $t_{n}, n=1,2,3, \ldots$ Then, $p(Q, t)$ and $b(Q, t)$ can be approximated by using a set of interpolation functions as illustrated below,

$$
\begin{aligned}
& p(Q, t)=\sum_{j=1}^{J} \sum_{m=1}^{N} \varphi^{m}(t) \eta_{j}(Q) p\left(Q_{j}, t_{m}\right) \\
& b(Q, t)=\sum_{j=1}^{J} \sum_{m=1}^{N} \theta^{m}(t) \zeta_{j}(Q) b\left(Q_{j}, t_{m}\right)
\end{aligned}
$$

where $m$ and $j$ refer to the time and space variations respectively, $\varphi^{m}, \theta^{m}, \eta_{j}$, and $\zeta_{j}$ are the interpolation functions, expressed as

$$
\begin{gathered}
\varphi^{m}(\tau)=\left\{\begin{array}{ll}
\left(\tau-t_{m-1}\right) / \Delta t & t_{m-1}<\tau \leq t_{m} \\
\left(t_{m+1}-\tau\right) / \Delta t & t_{m}<\tau \leq t_{m+1} \\
0 & \text { otherwise }
\end{array}, \theta^{m}(\tau)= \begin{cases}1 & t_{m-1} \leq \tau \leq t_{m} \\
0 & \text { otherwise }\end{cases} \right. \\
\eta_{j}(\xi)=\zeta_{j}(\xi)= \begin{cases}\left(\xi_{p}+1\right) / 2 & Q \in e_{p} \\
-\left(\xi_{q}-1\right) / 2 & Q \in e_{q} \\
0 & \text { otherwise }\end{cases}
\end{gathered}
$$

where $\xi$ is the coordinate along the boundary element, and $e_{p}$ and $e_{q}$ are the adjacent elements with a common node.

Substituting (15) into (13), we have

$$
c p_{i}^{n}+\sum_{j=1}^{J} \sum_{m=1}^{N} E_{i j}^{n m} p_{j}^{m}-\sum_{j=1}^{J} \sum_{m=1}^{N} D_{i j}^{n m} b_{j}^{m}=s_{i}^{n}
$$

where $n$ and $i$ refer to the time and space variations respectively, and $E_{i j}^{n m}=\int_{\Pi} \eta_{j}(Q) Y^{n m} \partial R / \partial n \mathrm{~d} \Pi, D_{i j}^{n m}=\int_{\Pi} \zeta_{j}(Q) T^{n m} \mathrm{~d} \Pi$

$$
Y^{n m}=\int_{0}^{t_{n}}\left[\phi^{m}(\tau) B+G / c_{0} \partial \phi^{m}(\tau) / \partial \tau\right] \mathrm{d} \tau, \quad T^{n m}=\int_{0}^{t_{n}} \theta^{m}(\tau) G \mathrm{~d} \tau .
$$

The values of $Y^{n m}$ and $T^{n m}$ depend on the positions of the sound wave front, as shown in the Table 2, where $A_{1,2}=\sqrt{c_{0}\left(t_{n}-t_{m}\right) \mp R}, A_{3,4}=\sqrt{c_{0}\left(t_{n}-t_{m+1}\right) \mp R}, A_{5}=c_{0}\left(t_{n}-t_{m}\right)$, $A_{6}=c_{0}\left(t_{n}-t_{m+1}\right), t_{r}=t_{n}-R / c_{0}$.

TABLE 2. Time integrated kernels.

\begin{tabular}{cccc}
\hline \multicolumn{1}{c}{ Cases } & $Y^{n m}$ & $T^{n m}$ \\
\hline $1:$ & $t_{m+1}<t_{r}$ & $\frac{1}{2 \pi c_{0} \Delta t}\left(\frac{A_{4}}{A_{2}}-\frac{A_{2}}{A_{4}}\right) \frac{2 c_{0} \Delta t}{A_{1} A_{4}+A_{2} A_{3}}$ & $-\frac{1}{2 \pi} \ln \left(\frac{A_{3} A_{4}+A_{6}}{A_{1} A_{2}+A_{5}}\right)$ \\
$2: \begin{array}{l}t_{m+1} \leq t_{n}, \\
t_{m}<t_{r} \leq t_{m+1}\end{array}$ & $\frac{1}{2 \pi c_{0} \Delta t}\left[\frac{A_{1}\left(A_{4}\right)^{2}}{A_{2} R}-\frac{A_{1} A_{2}}{R}\right]$ & $-\frac{1}{2 \pi} \ln \left(\frac{R}{A_{1} A_{2}+A_{5}}\right)$ \\
$3:$ & $t_{r}<t_{m}$ & 0 & 0 \\
\hline
\end{tabular}

The Gauss quadrature is employed to implement the space integration in $E_{i j}^{n m}$ and $D_{i j}^{n m}$. Besides, the instability problem in TDBEM is simply overcome by properly choosing the timestep and element length for convenience in the present model. The detailed process may be inferred to the work of Mansur and Brebbia (1982).

\section{Sound pressure radiated from vortex shedding}

In the flow filed solution, we have obtained the positions, circulations and velocities of all the vortices; therefore, it is convenient to evaluate the sound pressure through (9) and (10). However, it is a difficult task to solve these integral equations through pure numerical methods due to the "tail effect" of the 2D Green's function in the time domain, and some analytical operations is adopted.

we can readily find that the time integral in (10) has the same form with that of the second term on the right-hand side of (13), which is expressed as $\int_{0}^{t}\left(B+G / c_{0} \partial / \partial \tau\right) d \tau$. In this respect, (10) can be semi-analytically solved with the same discretization procedure in the time integration of TDBEM. The sound pressure radiated from the vortex can be approximated by using linear interpolation function, which is written as

$$
s_{i}^{n}=\sum_{m=1}^{N} Y^{n m} X^{m} .
$$

\section{RESULTS AND DISCUSSION}

To verify the validity of present model, the dimensions of the calculation domain and the position of sound pressure monitoring point are referred to the previous experimental work of Mohany and Ziada (2011), as shown in Fig.2. The circular cylinder with a diameter of $0.0158 \mathrm{~m}$ is placed on the centreline of a flow duct with dimensions of $L=1.016 \mathrm{~m}$ in length and $H=0.254 \mathrm{~m}$ in height. The range of the incoming velocity varies from $40 \mathrm{~ms}^{-1}$ to $75 \mathrm{~ms}^{-1}$. Numerous preliminary runs have been operated to determine the suitable values of timestep $\Delta t$ and the length of boundary element $l_{\mathrm{e}}$ to obtain 
the flow and sound field results simultaneously and stably. It is found that the following combination of parameters, $\Delta t=0.08 a / U, l_{\mathrm{e}}=0.01$, gives rise to stable results with enough accuracy and acceptable time expense. The rigid wall boundary conditions are applied on the duct wall. As the TDBEM satisfies the far field radiation condition automatically, there is no need to apply non-reflection boundary conditions on the duct ends, where the sound wave can freely release out of the duct.

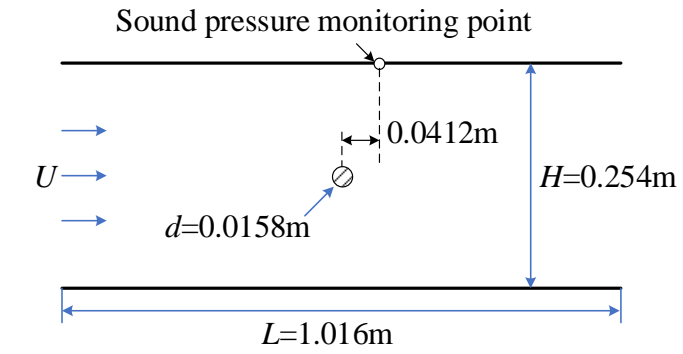

\section{Figure 2. Sketch of the Flow Duct Containing a Circular Cylinder.}

\section{Flow field results}

Only the flow field is calculated in this section, and 34000 steps are implemented, which require about 3 CPU hours on a personal PC. The last 32768 steps are analysed using fast Fourier transformation (FFT) with the former 1232 steps being discarded as transient (the flow is fully developed after about 900 timesteps).

As shown in Fig.3, the free vortex shedding frequency raises linearly with the increasing flow velocity, and the Strouhal number of the vortex shedding is about 0.2 , and the RMS value of $C_{1}$ at $U=54 \mathrm{~ms}^{-1}$ is 0.57 . A typical distribution of the flow pattern behind the cylinder can be found in Fig. 4, where the vortex street is clearly visible. The agreement between these results and the experimental data measured by Mohany and Ziada (2011) is reasonably good.
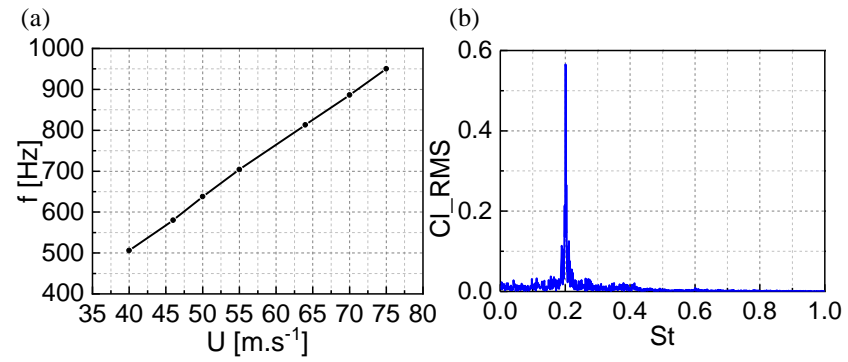

Figure 3. Flow Field Results: (a) dependence of Free Vortex Shedding Frequency on Flow Velocity, (b) the Spectrum of Lift Coefficient, $U=54 \mathrm{~ms}^{-1}$.

\section{Sound field results}

Both the flow field and sound field are calculated in this section. In view of the time expense of sound field solution, 17500 steps are implemented in each case and it costs about 30 CPU hours. Among those steps, the last 16384 steps with a total length about $0.2 \mathrm{~s}$ are analyzed using FFT, and the former 1116 steps are discarded as transient. Consequently, the frequency resolution is about $5 \mathrm{~Hz}$, which is not adequate for localizing the accurate resonant peaks of the spectra, therefore an interpolation correction method is introduced as compensation (Xie and Ding, 1996).

Fig. 4 gives the sound field distribution within the duct at a velocity of $54 \mathrm{~ms}^{-1}$. The Karman vortex street can be easily detected, as illustrated by the black dots in Fig.4. The high sound pressure region appears regularly in the middle region of the duct, where the sound pressure gets to maxima with antiphase at the positions on the duct wall just above and below the cylinder and decreases rapidly in both the upstream and downstream directions. This is a typical sound pressure pattern of the $\beta$-mode that relates to the first transverse acoustic mode of the duct.

Dependence of resonant SPL and frequency on the flow velocity is shown in Fig.5. With the velocity increasing from $40 \mathrm{~ms}^{-1}$ to $49 \mathrm{~ms}^{-1}$, the sound frequency keeps identical with the free vortex shedding frequency, which shows the same tendency with the case in free space. However, when the flow speed reaches $50 \mathrm{~ms}^{-1}$ the dominant frequency suddenly jumps up to the $\beta$-mode frequency of the duct, about $685 \mathrm{~Hz}$, and keeps nearly constant with the increasing of the flow velocity, until the velocity gets larger than $62 \mathrm{~ms}^{-1}$. This is a remarkable lock-in regime, which is recognized as the most typical characteristic of acoustic resonance. And as far as the authors know, this is the first physical model that successfully depicts the lock-in phenomenon of acoustic resonance induced by the cylinder wake. As shown in Fig. 5 (b), the predicted frequency of sound pressure in the duct agrees well with the experimental data qualitatively and quantitatively, although the width of the lock-in region is under-predicted, where the predicted upper flow velocity boundary is slightly lower than the measured data.

Fig 5(a) shows the variation of resonant SPL with the increasing flow velocity. In general, a humped sound pressure curve is observed, and the peak value appears near the middle of the lock-in region. With the velocity raising from $40 \mathrm{~ms}^{-1}$ to $55 \mathrm{~ms}^{-1}$, the corresponding sound pressure goes up sharply from $129 \mathrm{~dB}$ to $159.3 \mathrm{~dB}$, and it drastically falls back to $135.9 \mathrm{~dB}$ at $60 \mathrm{~ms}^{-1}$. After that, a second humped portion appears with a much lower peak. The present model gives an identical tendency of the SPL curve with the experimental data, but over-predicts the value of SPL by about $10 \mathrm{~dB}$. There are serval aspects may contribute to this discrepancy. Firstly, a 2D sound source model is used in this study, which may lead to higher SPL inherently. Secondly, the viscous dissipation of sound energy by the turbulence flow is not considered according to the non-viscous assumption, although the energy transfer from sound to flow field is involved by applying Kutta condition at the separation point. Thirdly, the vibration of the duct walls is not considered in this model, which is recognized as the main path for the sound energy releasing from the inner duct during acoustic resonance (Welsh et al., 1984).

Another distinct difference between the simulated SPL and measured data is that the velocity exciting the strongest resonance is also under-predicted and the extent of velocity divergency is about $4 \mathrm{~ms}^{-1}$, which is similar to that of the predicted lock-in region results in Fig.5(a). The most probably reason is that the boundary layer covering the 
cylinder is not calculated in this model, therefore the receptivity of sound wave, which physically decides the feedback process, cannot be accurately described.

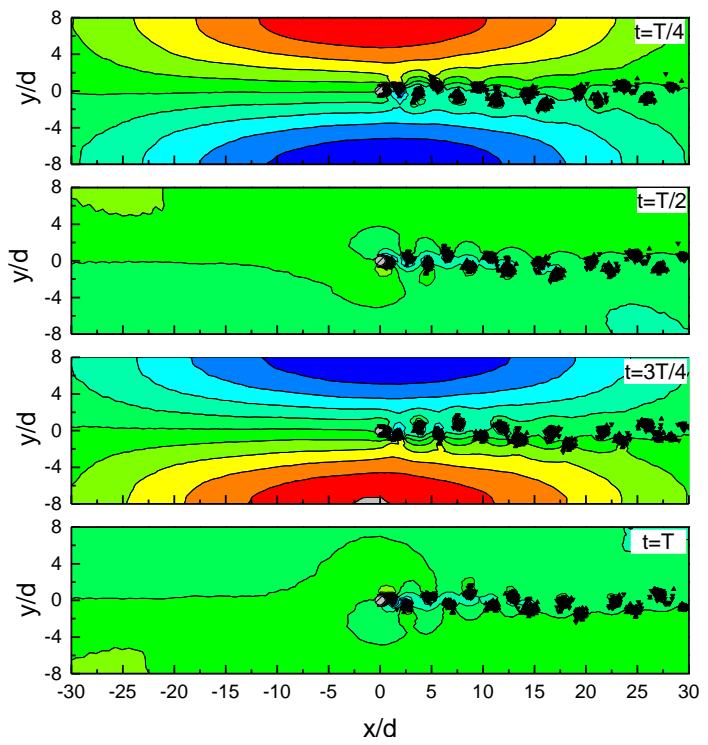

Figure 4. Periodic vortex shedding and Sound Field within the Duct (10 Levels from $-2500 \mathrm{~Pa}$ to $2500 \mathrm{~Pa}$ ).
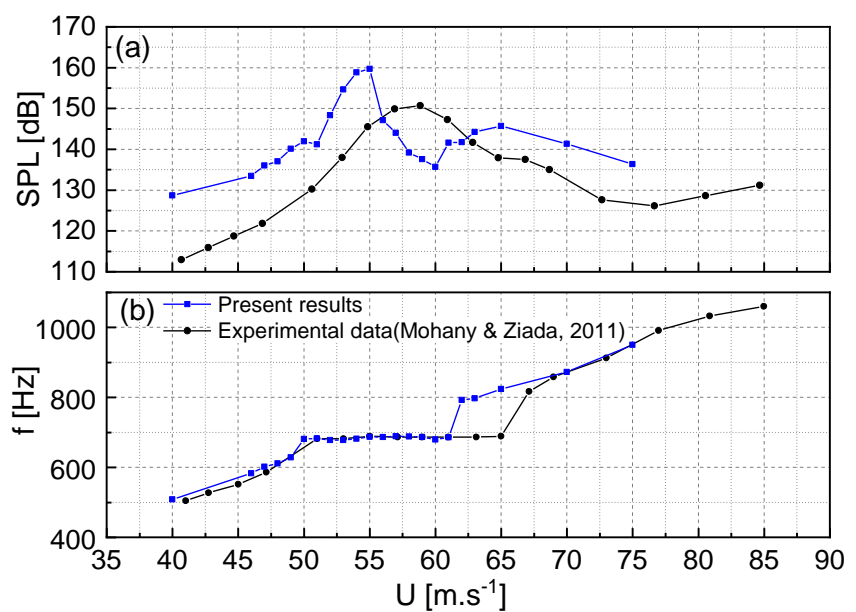

Figure 5. Variation of Resonant Frequency and SPL with Flow Velocity: (a) SPL, (b) Frequency

\section{Mechanism of the lock-in phenomenon}

As mentioned above, the lock-in phenomenon is an issue of interest in the acoustic resonance researches. In this part, some innovative results will be illustrated to deepen our understanding of the lock-in phenomenon. Some typical spectra of sound pressure on the duct wall (see Fig.2) are shown in Fig.6. Two dominant discrete frequency components co-exist in most cases: the one changing linearly with the incoming flow velocity is $f_{\mathrm{s}}$ and the other one remaining nearly constant around $685 \mathrm{~Hz}$ is $f_{\mathrm{a}}$. A vigorous competition exists between these two frequencies. When the flow velocity is far from the velocity extent of lock-in region, the SPL corresponding to $f_{\mathrm{s}}$ is higher than the other one, and vice versa. However, when the velocity is within $53 \mathrm{~ms}^{-1}$ to $55 \mathrm{~ms}^{-1}$, there is only one tone noise in the spectra. In addition, the variations of $f_{\mathrm{s}}$ and $f_{\mathrm{a}}$ with flow velocity are plotted in Fig. 7 for clarity. It is worth mentioning that, although the lock-in regime can be detected from $U=50 \mathrm{~ms}^{-1}$ to $U=61 \mathrm{~ms}^{-1}$, the synchronization of vortex shedding and sound wave can only be sustained in a narrow velocity range between $53 \mathrm{~ms}^{-1}$ to $55 \mathrm{~ms}^{-1}$, out of which the vortex shedding frequency differs from the $\beta$-mode frequency.

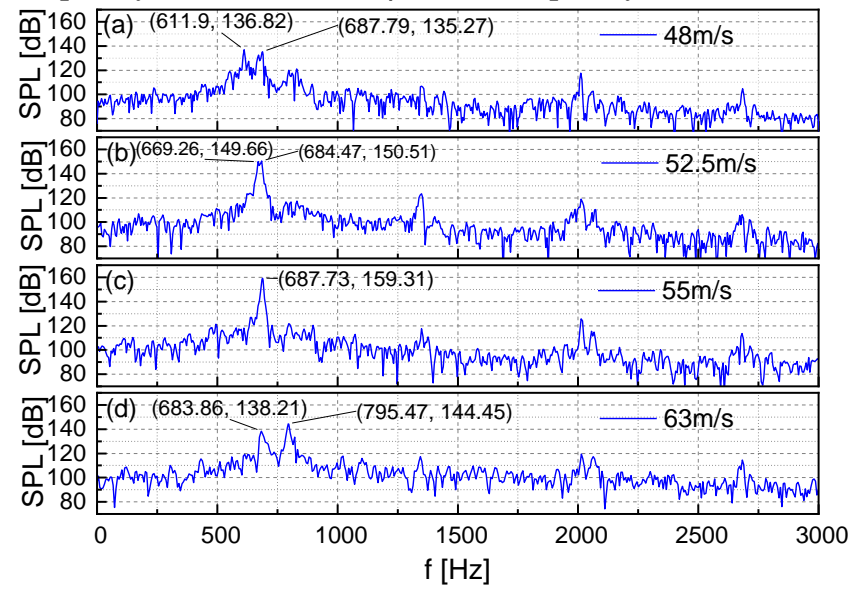

Figure 6. Sound Pressure Spectra at Various Velocities: (a) $48 \mathrm{~ms}^{-1}$, (b) $52.5 \mathrm{~ms}^{-1}$, (c) $55 \mathrm{~ms}^{-1}$, (d) $63 \mathrm{~ms}^{-1}$

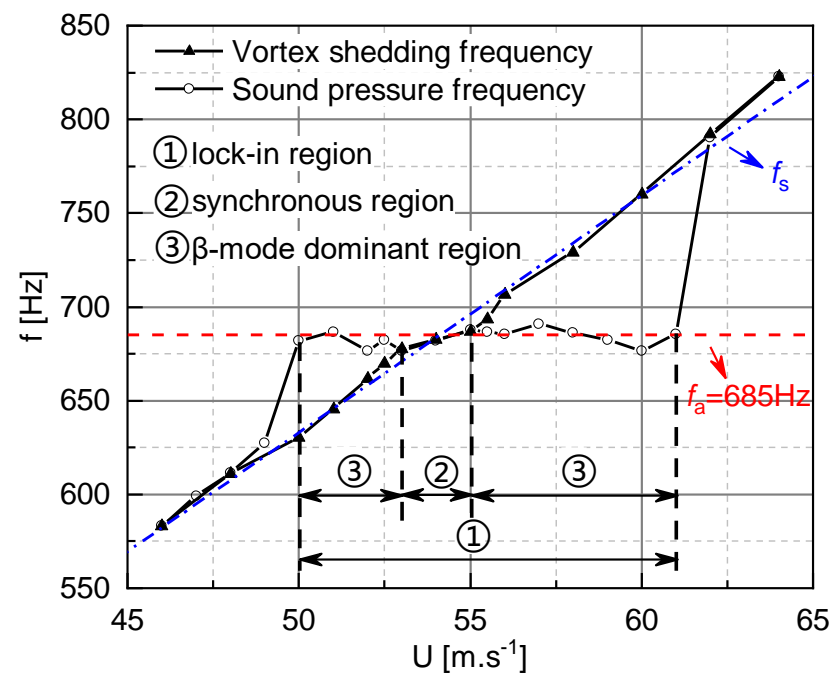

Figure 7. Frequencies of Vortex shedding and Resonant Sound Pressure with Acoustic Feedback

Actually, the flow duct can be recognized as a synchronous system (or feedback loop) with two oscillators (Pikovsky et al., 2001): one is the vortex shedding from the cylinder (wake mode) and the other is the $\beta$-mode sound wave within the duct (acoustic mode) and $u_{\mathrm{a}}$ is the coupling bridge between them. The forward portion of this loop is the acoustic response of the duct excited by the fluctuating vortex shedding where $f_{\mathrm{s}}$ and $f_{\mathrm{a}}$ co-exist. And when the mismatch between them is less than a threshold, the SPL of $\beta$-mode would become dominant in the spectrum. The feedback part of this loop involves the tuning effect of sound wave on vortex shedding through $u_{a}$. And when the two frequencies get close to each other, mutual adjustment (synchronization) would occur, giving rise to a common frequency between them as demonstrated in Fig.7. In this aspect, the lock-in regime can be divided into two patterns, the synchronous regime $\left(0.98<f_{\mathrm{s}} / f_{\mathrm{a}}<1.03\right)$ and the $\beta$-mode 
dominant regime $\left(0.93<f_{\mathrm{s}} / f_{\mathrm{a}}<0.98\right.$ and $\left.1.03<f_{\mathrm{s}} / f_{\mathrm{a}}<1.16\right)$, which is similar to character of vortex induced vibration (Zhang et al., 2015). There are two $\beta$-mode dominant regions on both sides of the synchronous region, and the right one is slightly wider than the left one. Furthermore, the strongest resonance appears at the end of synchronous region, which can be readily detected by combining Fig. 5 and Fig. 7.

As illustrated above, the extent of lock-in region mainly depends on the competition between $f_{s}$ and $f_{a}$. The quantity through which the coupling is made, $u_{\mathrm{a}}$, is dominated by the frequency mismatch between $f_{s}$ and $f_{a}$. This can be illustrated from the results in Fig. 8. We can see that the ratio of $u_{a}$ to $U$ is beyond $5 \%$, when the synchronization is sustained. This agrees well with the experimental measurement of Welsh et al (1984).

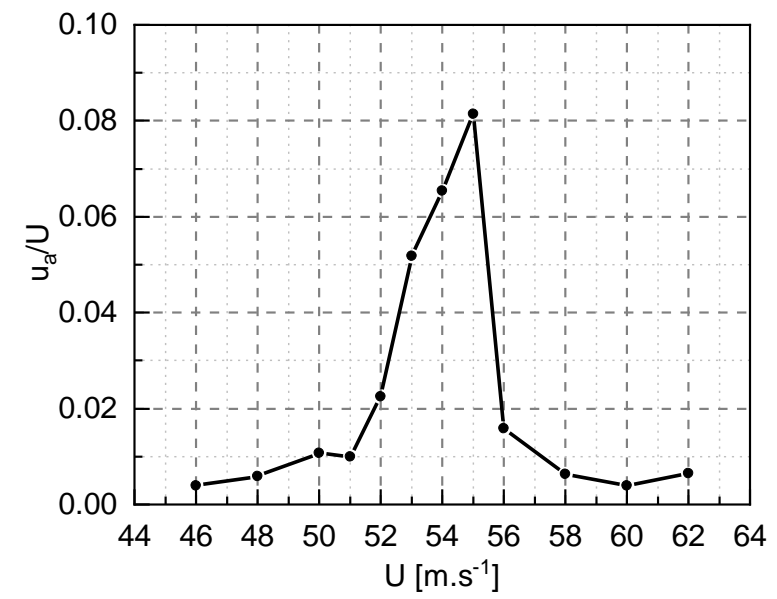

Figure 8. Velocity Ratio of Acoustic Particle and Incoming Flow

\section{CONCLUSIONS}

A 2D physical model to study the acoustic resonance induced by wake flow of a circular cylinder within a flow duct is established based on DVM for flow field, vortex sound analogy for sound radiation and TDBEM for sound propagation. This physical model is meshless and operated in the time-domain, which calculates the flow field, sound field and the coupling between them simultaneously, whose accuracy has been validated by previous experimental data. The typical characters of acoustic resonance including the lock-in phenomenon are well captured. The main conclusions are summarized as follows.

(1) The present model not only reasonably predict the distribution of vortex street behind the cylinder and the sound field within the duct but also accurately forecast the Strouhal number of the vortex free shedding and the inherent frequency of $\beta$-mode.

(2) The lock-in phenomenon is successfully captured by the present model, where the tendency of resonant frequency and SPL with the increasing flow velocity agree well with the experimental data, although the value of SPL is overpredicted by about $10 \mathrm{~dB}$ due to the $2 \mathrm{D}$ sound source model and ignoring of the sound energy dissipation.

(3) The flow duct containing a cylinder can be recognized as a synchronous system with two oscillators: the vortex shedding (wake mode) and the acoustic mode. The underlying mechanism of lock-in is the competition of between these two oscillators, and the extent of lock-in region is dominated by the acoustic mode.

(4) Two types of lock-in patterns, the synchronous regime and $\beta$-mode dominant regime, are identified depending on the frequency mismatch and coupling strength between the vortex shedding and acoustic $\beta$-mode. The synchronous regime is found when the $f_{\mathrm{s}}$ approaches $f_{\mathrm{a}}$ $\left(0.98<f_{\mathrm{s}} / f_{\mathrm{a}}<1.03\right)$, where the corresponding velocity ratio of $u_{a}$ to $U$ is beyond $5 \%$. Meanwhile, the $\beta$-mode dominant regime is detected when the frequency mismatch is slightly larger $\left(0.93<f_{\mathrm{s}} / f_{\mathrm{a}}<0.98\right.$ and $\left.1.03<f_{\mathrm{s}} / f_{\mathrm{a}}<1.16\right)$.

(5) The strongest resonance appears at the upper velocity boundary of the synchronous region, which is slightly underpredicted. The most probably reasons are the receptivity of boundary layer to the feedback sound is simplified by applying Kutta condition at the separation point, which would inevitably affect the quantitative accuracy of the present model and need further improvement.

\section{NOMENCLATURE}

\begin{tabular}{ll}
\multicolumn{2}{l}{ Variables: } \\
$a$ & Radius of cylinder \\
$A$ & A constant relates to the type of the incoming flow \\
$c$ & A constant related to the position of field point \\
$e_{p}, \quad e_{q}$ & Adjacent elements with a common node \\
$c_{0}$ & Velocity of sound wave \\
$f_{a}$ & Inherent frequency of $\beta$-mode \\
$f_{s}$ & Free vortex shedding frequency \\
$\mathrm{G}$ & 2D free space Green's function in the time domain \\
$\mathrm{H}$ & Heaviside function \\
$\mathbf{n}$ & Normal vector \\
$p$ & Pressure \\
$\mathrm{Q}$ & Position of field point \\
$s$ & Sound source radiated from the vortices \\
$t$ & Time \\
$u$ & Horizontal component of velocity \\
$u_{a}$ & Acoustic particle velocity \\
$\mathrm{U}$ & Flow velocity \\
$v$ & Vertical component of velocity \\
$\mathbf{v}$ & Convection velocity of vortex \\
$\mathbf{w}$ & Complex velocity \\
$\mathbf{x}$ & Position of observation point \\
$\mathbf{y}$ & Position of source point \\
$\mathbf{z}$ & Complex coordinate \\
\end{tabular}

\section{Subscripts:}

d Drag force

$i \quad$ i-th space variation

$j \quad \mathrm{j}$-th space variation

1 Lift force

$m \quad$ m-th time variation

$n \quad$ n-th point vortex

nv Nascent point vortex

$\mathrm{s} \quad$ Separation

\section{Superscripts:}

$m \quad$ m-th time variation 
n-th time variation

\section{Greek:}

$\Gamma \quad$ Strength of vortex

$\Lambda \quad$ Length of the feeding sheet

$\rho_{0} \quad$ Ambient far-field density

$\tau \quad$ Sound emission time

$\omega \quad$ Vorticity of a vortex

$\theta_{s} \quad$ Separation angle of shear layer

$\xi \quad$ Coordinate along the boundary element

$\Pi \quad$ Boundary of two-dimensional region

$\varphi^{m}, \theta^{m}, \eta_{j}, \zeta_{j} \quad$ Interpolation functions

\section{ACKNOWLEDGMENTS}

The authors acknowledge financial support from the NSFC, under grant 51806146.

\section{References}

Arthurs D., Bolduc M., Ritcey A., and Ziada S. (2013). Effect of Sound on the Wake of Side-by-Side Cylinders in a Duct. Proceedings of the ASME 2013 Pressure Vessels and Piping Conference. doi:10.1115/PVP2013-97021

Blevins R. D. (1984). Review of sound induced by vortex shedding from cylinders. Journal of Sound and Vibration 92 (4), 455-470. doi:10.1016/0022460x(84)90191-3

Davies M E. (1976). A comparison of the wake structure of a stationary and oscillating bluff body, using a conditional averaging technique. Journal of Fluid Mechanics 75 (02), 209-231. doi:10.1017/S0022112076000189

Hong Z., Dai X., Zhou N., Sun X., and Jing X. (2014). Suppression of Helmholtz resonance using inside acoustic liner. Journal of Sound and Vibration 333 (16), 3585-3597. doi:10.1016/j.jsv.2014.02.028

Karthik B., Chakravarthy S., and Sujith R. (2008). Mechanism of pipe-tone excitation by flow through an orifice in a duct. International Journal of Aeroacoustics 7 (34), 321-347. doi:10.1260/1475-472X.7.3.321

Katasonov M. M., Sung H. J., and Bardakhanov S. P. (2015). Wake flow-induced acoustic resonance around a long flat plate in a duct. Journal of Engineering Thermophysics 24 (1), 36-56. doi:10.1134/S1810232815010051

Kiya M., Sasaki K., and Arie M. (1982). Discrete-vortex simulation of a turbulent separation bubble. Journal of Fluid Mechanics 120, 219-244. doi:10.1017/S0022112082002742

Lewis R. I. (2005). Vortex element methods for fluid dynamic analysis of engineering systems (Vol. 1). Cambridge University Press.

Liu X., Willeke T., Herbst F., Yang J. and Sume J. (2018) A theory on the onset of acoustic resonance in a multistage compressor. Journal of Turbomachinery 140(8), 081003. doi:10.1115/1.4040551

Lotfy A., and Rockwell D. (1993). The near-wake of an oscillating trailing edge: mechanisms of periodic and aperiodic response. Journal of Fluid Mechanics 251 (1), 173201. doi:10.1017/S0022112093003374

Mansur W. J., and Brebbia C. A. (1982). Formulation of the boundary element method for transient problems governed by the scalar wave equation. Applied Mathematical Modelling, 6 (4), 307-311. doi:10.1016/S0307904X(82)80039-5

Mohany A., and Ziada S. (2009). Numerical Simulation of the Flow-Sound Interaction Mechanisms of a Single and Two-Tandem Cylinders in Cross-Flow. Journal of Pressure Vessel Technology 131 (3), 031306. doi:10.1115/1.3110029

Mohany A., and Ziada S. (2011). Measurements of the dynamic lift force acting on a circular cylinder in cross-flow and exposed to acoustic resonance. Journal of Fluids and Structures 27 (8). 1149-1164.

Mohany A. (2012). Flow-Excited Acoustic Resonance of Multiple Isolated Cylinders in Cross-Flow. Aecl Nuclear Review 1 (1), 45-55. doi:10.12943/ANR.2012.00007

Mohany A., Arthurs D., Bolduc M., Hassan M., and Ziada S. (2014). Numerical and experimental investigation of flow-acoustic resonance of side-by-side cylinders in a duct. Journal of Fluids and Structures 48, 316-331. doi:10.1016/j.jfluidstructs.2014.03.015

Pikovsky A., Rosenblum M., and Kurths J. (2002). Synchronization: a universal concept in nonlinear science. Cambridge University Press.

Parker R. (1968). An investigation of acoustic resonance effects in an axial flow compressor stage. Journal of Sound and Vibration 8 (2), 281-297. doi:10.1016/0022460X(68)90233-2

Powell A. (1964). Theory of vortex sound. The Journal of the Acoustical Society of America 36 (1), 177-195. doi:10.1121/1.1918931

Sarpkaya T., and Schoaff R. L. (1979). Inviscid model of two-dimensional vortex shedding by a circular cylinder. AIAA Journal 17, 1193-1200. doi:10.2514/3.61300

Shaaban M., and Mohany A. (2018). Flow-induced vibration of three unevenly spaced in-line cylinders in crossflow. Journal of Fluids and Structures 76, 367-383. doi:0.1016/j.jfluidstructs.2017.10.007

Tan B. T., Thompson M., C., and Hourigan K. (2004). Flow past rectangular cylinders: receptivity to transverse forcing. Journal of Fluid Mechanics 515, 33-62. doi:10.1017/S0022112004000242

Welsh M. C., Stokes A. N., and Parker R. (1984). Flowresonant sound interaction in a duct containing a plate, Part I: semi-circular leading edge 95 (3), 305-323. doi:10.1016/0022-460x(84)90670-9

Xie M., and Ding K. (1996). Corrections for frequency, amplitude and phase in a fast Fourier transform of a harmonic signal. Mechanical Systems and Signal Processing 10 (2), 211-221. doi:10.1006/mssp.1996.0015

Yokoyama H., Kitamiya K., and Iida, A. (2013). Flows around a cascade of flat plates with acoustic resonance. Physics of Fluids 25 (10), 106104. doi:10.1063/1.4825376

Zhang W., Li X., Ye Z., and Jiang Y. (2015). Mechanism of frequency lock-in in vortex-induced vibrations at low Reynolds numbers. Journal of Fluid Mechanics 783, 72-102. doi:10.1017/jfm.2015.548 